\title{
Cloud computing en la industria financiera
}

\author{
Gastón Bruno ${ }^{1}$
}

\section{Resumen}

Cloud computing continúa en constante crecimiento durante los últimos años. Esto es consecuencia de los beneficios que brinda, principalmente en términos económicos y de administración para aquellas compañías que lo adopten. Debido a una de sus principales características de compartimiento de recursos para aprovechar al máximo todas las capacidades, el esquema de cloud computing es bastante extenso y permite distintas configuraciones. Esto permite que no se limite únicamente a grandes compañías, pudiendo ofrecer soluciones inclusive a pequeños consumidores. Pero esta característica de "hacer público lo privado" genera controversias principalmente en cuanto a los aspectos de seguridad y privacidad de la información. Es por esto, que si bien los pequeños consumidores se están acercando a este esquema mirando los beneficios que obtienen, las compañías de la industria financiera no logran todavía obtener un pleno convencimiento para implementar el esquema de cloud computing. Este trabajo comienza describiendo el modelo de cloud computing, lo que llevará a plantear sus beneficios y potenciales inconvenientes que puede generar bajo un modelo de adopción en la industria financiera. Finalmente se plantearán recomendaciones en base a lo desarrollado para establecer una guía de adopción.

Palabras Clave: Banca, Computación en la Nube, Sistemas de Información Distribuidos, Servicios en Línea, Arquitectura de Software. 


\begin{abstract}
In recent years, Cloud computing continues to grow steadily. This is mainly due to the economic and administrative benefits it provides companies with. Because one of the main characteristics of resource sharing to maximize all the capabilities, cloud computing scheme is quite extensive and allows various configurations. This allows offering solution not only to large companies, but also to small consumers. But this feature of "making public what is private" is controversial mainly in terms of data security and privacy. Because of this, while small consumers are approaching this scheme looking for benefits, the financial industry companies still fail to get a complete certainty to implement the cloud computing scheme. This paper begins by describing the cloud computing model, raising its benefits and potential drawbacks that may appear during an adoption model in the financial industry. Finally, we will present recommendations based on what was developed to establish an adoption guide.
\end{abstract}

Keywords: Banking, Cloud Computing, Distributed Information Systems, Online Services, Software Architecture. 


\section{Adopción de cloud computing}

Varias consultoras especializadas en estudios de mercado han analizado el movimiento creciente de servicios de cloud computing pronosticando para los próximos tres años un mercado del orden de cientos de miles de millones de dólares. (Gartner, 2010), (Gartner, 2008), (Gartner, 2009), (Gartner, 2010), (Gartner, 2011), (Analysys Mason, 2010), (IDC, 2010), (WinterGreen Research, 2010). Por ejemplo, Global Industry Analysts, Inc. indicó que el mercado de servicios de cloud computing alcanzará los U\$D 222.500 millones en el año 2015 (Global Industry Analysts, 2011).

En lo que respecta puntualmente a la industria financiera, existen estudios de mercado que reflejan su creciente tendencia de adopción manifestando que del $20 \%$ de estas empresas ya han implementado y están usando cloud computing (IDC, 2011). Además IDC demostró que esta industria está más adelantada en cuanto a la adopción de cloud computing en comparación con las demás (IDC, 2011).

Si bien es posible analizar varios aspectos de cloud computing, el más relevante en este trabajo es el relativo a la seguridad y sus riesgos. En relación a esto, resulta interesante observar dónde se ubica esta cuestión en el estudio anual de Gartner acerca del grado de adopción de cloud computing (Gartner, 2011).

En julio de 2011, Gartner indicó que "Cloud Security" se encuentra casi en el pico de su modelo "Hype Cycle" (Gartner, 2011), lo que indica que se está iniciando un proceso de generación de documentación al respecto, ya sea que luego se termine adoptando o no. En este sentido, Jay Heiser menciona que existen cuatro compañías que están generando modelos de trabajo estandarizados para que los consumidores de cloud computing evalúen a sus proveedores en los aspectos relativos a la seguridad. Se necesita un tiempo prudencial para que estas metodologías sean ejecutadas y evaluadas, por lo que llevará de cinco a diez años que esto tenga el peso suficiente como para ser tomado en cuenta como material que posea un valor considerable para su adopción.

\section{Conceptos de cloud computing en relación con la industria financiera}

Cloud computing es una práctica que contempla el acceso en demanda a hardware, software y servicios de un tercero por medio de internet con el objetivo de reducir costos de infraestructura. Bajo una perspectiva global a nivel empresarial, las entidades financieras que adopten cloud computing no solo podrán reducir costos en el área de tecnología sino que, podrán además enfocar sus procesos y recursos en profundizar sus actividades de negocio orientados a maximizar la cartera de productos ofrecidos a sus clientes actuales (Potenciar Cross-Selling y Up-Selling 
en instituciones bancarias (Salesforce.com, 2011), (Microsoft, 2011)) y además a captar otros nuevos. Tener una capacidad tecnológica ágil y flexible con capacidad de responder adecuadamente a las demandas del negocio favorecerá la alineación del área de tecnología informática (T.I.) con el negocio, generando una ventaja competitiva que acelere el aumento de rentabilidad.

El Instituto de Estándares y Tecnología (NIST) define cloud computing como un modelo para habilitar acceso ubicuo, conveniente y bajo demanda por medio de la red a un pool compartido de recursos computacionales configurables que pueden ser rápidamente aprovisionados y liberados con un esfuerzo mínimo de administración o de interacción del proveedor (NIST, 2011).

\section{Características esenciales}

Auto servicio bajo demanda. Un consumidor puede proveer capacidades computacionales unilateralmente, automáticamente según se necesita y sin requerir interacción humana con cada proveedor de servicio.

Amplio acceso de red. Las capacidades están disponibles por medio de la red y son accedidas a través de mecanismos estándares que promueven el uso de plataformas heterogéneas de clientes delgados o gruesos.

Agrupación de recursos. Los recursos computacionales del proveedor se reúnen para servir a múltiples consumidores utilizando un modelo de múltiples niveles, con diferentes recursos físicos y virtuales asignados de forma dinámica y reasignados de acuerdo a la demanda del consumidor.

Elasticidad rápida. Las capacidades pueden ser rápida y elásticamente aprovisionadas, hasta en algunos casos automáticamente, para escalar horizontalmente y ser liberadas rápidamente para escalar verticalmente.

Servicio medido. Los sistemas de nube automáticamente controlan y optimizan el uso de recursos mediante el aprovechamiento de una capacidad de medición a un cierto nivel de abstracción adecuado para el tipo de servicio.

\section{Modelos de servicio}

Con los diferentes tipos de oferta de cloud computing existen distintas opciones en cuanto a cuáles servicios se podrán llevar a la nube y cuáles otros permanecerán en sitio. Los puntos fuertes que influenciarán a las entidades financieras a tomar esta decisión son las prioridades y requerimientos de seguridad.

Software as a Service (SaaS). La capacidad provista al consumidor es la de usar las aplicaciones del proveedor ejecutándose en una infraestructura de cloud.

Es común para las entidades financieras que cuenten con una red de modelos 
de negocio que se encuentren estrechamente conectados entre sí. Para esto, SaaS provee distintas opciones de entrega:

- Experiencia del usuario consistente.

- Arquitectura y modelo de datos común.

- Flexibilidad al poder satisfacer de manera simple los requerimientos complejos.

- Adaptabilidad al permitir cambios en cualquier punto del tiempo.

Platform as a Service (PaaS). La capacidad provista al consumidor es la de desplegar en la infraestructura de cloud aplicaciones creadas por el mismo consumidor o adquiridas, creadas usando un lenguaje de programación y herramientas soportadas por el proveedor.

Otra característica común en las compañías de la industria financiera es la de poseer sistemas propietarios y/o antiguos que las limitan en ciertas oportunidades en el momento de desarrollar nuevos proyectos. La arquitectura abierta de PaaS rompe con este tipo de limitaciones ya que soporta la integración con aplicaciones legacy a la vez que ofrece interoperabilidad con los sistemas que se encuentren en sitio.

Infrastructure as a Service (IaaS). La capacidad provista al consumidor es la de aprovisionar procesamiento, almacenamiento, redes y otros recursos computacionales fundamentales para los cuales el consumidor es capaz de desplegarlos y ejecutar arbitrariamente software, que puede incluir sistemas operativos y aplicaciones.

En cuanto a los costos, la ventaja que ofrece IaaS es la posibilidad de ajustar la granularidad de costos de infraestructura al máximo nivel ya que es un modelo de centro de datos en demanda que provee recursos computacionales basados en los recursos que se consumen. De esta manera se paga solamente por lo que se consume.

En cuanto a la disponibilidad, siendo que este esquema de cloud computing ofrece almacenamiento, redes y servidores como servicio; se entregan virtualmente recursos computacionales en las manos de hasta las entidades financieras más pequeñas para cumplir con sus picos de demanda. En este sentido, dichas entidades pueden reducir el time-to-market. Además esto reduce el TCO ya que bajo el modelo de alquiler, el costo total de la propiedad de la infraestructura no será asociada a los consumidores.

\section{Modelos de despliegue}

Cloud privado. La infraestructura de cloud es operada únicamente para una organización.

Cloud comunitario. La infraestructura de cloud es compartida por varias 
organizaciones y soporta una comunidad específica que contiene preocupaciones compartidas.

Cloud público. La infraestructura de cloud es puesta a disponibilidad del público en general o a un largo grupo industrial y es propiedad de una organización que vende servicios de cloud.

Cloud híbrido. La infraestructura de cloud es una composición de dos o más modelos de despliegue de cloud que permanecen como entidades únicas, pero son unidas entre sí por medio de una tecnología estandarizada o propietaria que permite portabilidad de datos y aplicación.

\section{Implicancias positivas en la adopción de cloud computing para las entidades financieras}

Aquellas entidades financieras que se animen a aventurarse en los modelos de cloud computing tendrán la posibilidad de redefinir sus estrategias y en consecuencia, sus operatorias para llevarlas a cabo.

\section{Crecimiento gradual}

La existencia de estos diferentes modelos genera una de las ventajas para las entidades financieras al permitir que éstas puedan incorporarse al esquema de cloud computing de manera gradual (IDC, 2010).

\section{Aspectos económicos}

En lo que respecta al cloud privado, IDC ha clasificado las principales razones por las cuales las entidades financieras deberían adoptar este modelo (IDC, 2011).

Claridad para establecer mediciones de costos. La posibilidad de obtener un modelo más transparente para cobrar los servicios a los usuarios.

Reducción de costos de infraestructura. Se logra mediante el compartimiento de recursos a lo largo de diferentes áreas de negocios y la estandarización de procesos.

Pago por uso. IDC ha realizado también esta clasificación para el cloud público, resultando dentro de las principales razones los bajos montos de pago mensual y asociado a esto, la posibilidad de pagar solamente por lo que se requiere (IDC, 2011).

De CAPEX hacia OPEX. Adoptar un esquema de cloud computing permite generar una transición de un modelo de inversión de capital hacia el modelo gestión de administración operacional.

Economías de escala. Al ofrecer un sitio de infraestructura tecnológica centralizado, cloud computing aprovecha el concepto de economías de escala (Microsoft, 2010) para reducir los costos de los elementos de hardware, software y su administración.

Eficiencia operativa. IDC ha clasificado los beneficios económicos que resultan de la adopción de cloud computing, resultando dentro de los principales la reducción 
del gasto total de hardware, software y mantenimiento (IDC, 2011). En este mismo estudio, IDC ha identificado otra ventaja en relación a la capacidad de maximizar la utilización de los recursos mediante esquemas de virtualización, ofreciendo un esquema eficiente en costos.

\section{Agilidad}

Las soluciones de cloud computing basadas en internet permiten mayor flexibilidad, agilidad y capacidad de escalar hacia arriba o hacia abajo en la medida que la demanda del negocio lo disponga (IDC, 2011). Una encuesta realizada por Gartner indica que el $55 \%$ de las empresas encuestadas ve a la agilidad y velocidad como los principales impulsadores para moverse hacia esquemas de cloud (Gartner, 2011).

\section{Productividad}

La integración de aplicaciones en tiempo real permite a los agentes de marketing y ventas obtener la información que necesitan al instante, de manera de ofrecer los productos más adecuados para cada cliente aprovechando cada comunicación entre ellos (IDC, 2011).

\section{Reducción del go-to-market}

La disposición instantánea de recursos de infraestructura que soportan las nuevas ideas de marketing es un habilitador fundamental en la reducción de tiempos en nuevas campañas y/o servicios. Esto permite un diferenciamiento entre los competidores que resulta en otra ventaja competitiva (IDC, 2011).

\section{Nuevas formas de generar negocios}

Según K. P. Shashidharan estos servicios emergentes de cloud computing apuntan a mejorar la productividad facilitando la colaboración en tiempo real, creando oportunidades de presencia institucional en cualquier lado (Shashidharan, 2011).

Mejoras en el nivel de servicio hacia el cliente. La estrecha relación de los servicios de cloud computing con las herramientas de relacionamiento social en auge permite a las entidades financieras a enfocarse y conocer aún mejor a sus clientes. Esto no solo redunda en poder ampliar la cantidad de productos vendidos a un mismo cliente, sino también en poder captar nuevos clientes.

Banca móvil. Otra característica que habilita cloud computing es la posibilidad de la implementación de un modelo de Mobile Banking (IDC, 2011). Esta implementación no solo actúa como un diferenciador en comparación con los otros bancos, sino que además aumenta la calidad de servicio a los clientes.

\section{Integración}

Si bien cada entidad financiera se vale por sí misma para ofrecer sus productos y se intenta diferenciar de sus competidores para obtener una ventaja competitiva, en la capa más profunda del sistema financiero todas estas entidades se encuentran comunicadas. Cloud computing permite establecer una plataforma comunitaria en la cual las entidades financieras puedan compartir recursos de tecnología y servicios bajo un esquema de regulación, conectividad y administración común (IDC, 2011). 


\section{Green IT}

La consolidación de infraestructura tecnológica en sitios de proveedores de cloud computing especialmente preparados para esto, permite un aprovechamiento eficiente de la energía, ya sea para alimentación o refrigeración.

\section{Más foco en el negocio}

Cloud computing permite al departamento de T.I. pasar de un esquema de tareas operativas a otras del tipo tácticas. Liberándose las tareas de administración de parches, mantenimiento y otras de este estilo, el departamento de T.I. puede capitalizar ese tiempo ganado en colaborar con la alta dirección en la generación de nuevas estrategias de negocio que aporten valor a la institución.

\section{Aspectos a tener en cuenta}

Si bien cloud computing ofrece varios beneficios, las entidades financieras verán que existen ciertos aspectos para los cuales se determina que quizás su adopción no es una solución que sea acorde para cada tipo de problema.

\section{Seguridad}

Algunos de los puntos que detienen a ciertas instituciones financieras a implementar cloud computing engloban a la seguridad en primer lugar, seguido por la privacidad, confidencialidad, integridad de los datos, requerimientos de autenticación, ubicación de los datos, disponibilidad y recuperación (IDC, 2011), (IDC, 2011).

Seguridad lógica. En lo relativo a la seguridad, IDC clasifica los diferentes niveles de riesgo para cada modelo de cloud computing (IDC, 2010). Establece que bajo el modelo de IaaS la entidad financiera es la responsable del control de acceso, mientras que el proveedor de servicios de cloud computing es el encargado de proveer seguridad perimetral y encriptación de datos. Para el esquema de PaaS el esquema es similar, mientras que bajo SaaS el proveedor de servicios de cloud computing tiene una mayor responsabilidad al tener que separar y proteger los datos de las diferentes entidades financieras, proveyendo para esto herramientas de autenticación y cumpliendo con las regulaciones y políticas de cada entidad financiera.

Para el NIST las responsabilidades bajo el aspecto de seguridad bajo un esquema de cloud computing son compartidas entre el proveedor y la institución financiera que consuma dichos servicios (NIST, 2011).

Proliferación de los datos. Christian Verstraete menciona en su trabajo acerca de los datos en el esquema de cloud computing tres aspectos a tener en cuenta en cuanto a la seguridad de los datos (Verstraete, 2011).

Para brindar tolerancia a fallos, los esquemas de cloud computing pueden duplicar la información en sitios distintos, generando una proliferación de la información de las entidades financieras. Estas compañías, que si bien son las 
dueñas de la información, pierden el control. Por lo tanto, si este aspecto no se tiene en consideración con anticipación, puede que se violen algunas regulaciones gubernamentales y/o peor aún, se potencie el nivel de riesgo de la exposición de su información. Otras cuestiones relacionadas a esto surgen de evaluar las condiciones de respaldo de datos. Es posible que el proveedor de cloud computing utilice servicios de terceros para esto, lo que lleva a preguntarse por ejemplo: ¿A quién le pertenece en este caso la información en las cintas?, ¿Dónde guarda el proveedor de servicios de cloud computing las cintas?, ¿Si se borra la información en un sitio, se borra automáticamente en todos los demás sitios?

Asociado a esto último surge otro interrogante en cuanto a la eliminación de la información. Cuando se elimina información, ¿Se emplean técnicas de blanqueo del medio o es posible que el próximo usuario recupere la información del volumen de datos?

Seguridad física. El proveedor de servicios de cloud computing no solamente debe asegurarse de proveer herramientas de protección de acceso no autorizado a los datos a nivel de red, entre aplicaciones o instancias de sistema operativo; sino que además debe garantizar la protección física de los datos.

\section{Privacidad}

Cada país tiene diferentes regulaciones en cuanto a dónde está permitido que resida información de sus ciudadanos. Por ejemplo, para aquellos que pertenecen a la Unión Europea se le prohíbe que se transmitan ciertos datos personales hacia otros países que no cumplan con los mismos niveles de protección de datos (Protection of personal data, 2011). El hecho de que cloud computing ofrezca un modelo computacional que atraviese fronteras puede llegar a ser un problema para ciertas instituciones financieras que se vean afectadas por regulaciones como éstas. Dónde reside la información debe ser un aspecto minuciosamente observado por las instituciones financieras en los contratos con los proveedores de cloud computing. La jurisdicción legal de los datos es definida por las leyes del país en donde residen los datos, lo que puede concluir en algunas implicancias legales.

Propiedad intelectual

Existe en ciertos casos la disyuntiva acerca de si los datos le pertenecen al proveedor de cloud computing o a la entidad financiera que consuma sus servicios.

\section{Regulaciones}

Para este mercado financiero, las regulaciones no solamente son importantes, sino que además se concentran bajo un contexto dinámico. Por esto, cualquier iniciativa que mueva a las entidades financieras a establecer un cambio en su modelo operativo, debe tener la flexibilidad necesaria para adaptarse a estas cambiantes regulaciones.

\section{Auditoría}

Bajo el marco de la importancia de las regulaciones y la privacidad de la información resulta importante establecer políticas de auditoría que faciliten la resolución de problemas en el momento que sea necesario realizar investigaciones. 


\section{Modelos contractuales}

Las condiciones contractuales entre el proveedor de servicios de cloud computing y las entidades financieras presentan otro aspecto importante a tener en cuenta.

Garantía de nivel de servicio. Dentro de los contratos se denota la importancia de los niveles de servicio para los cuales el proveedor de servicios de cloud computing garantiza un determinado nivel de servicio, disponibilidad, tiempo de respuesta, capacidad de recursos, etc. Esta misma consideración se debe tener en cuenta con el proveedor del enlace de red.

Flexibilidad en los contratos. Resulta importante dejar en claro las pautas de terminación de contrato de manera que se pueda cambiar rápidamente y $\sin$ problemas de proveedor de servicios si así lo necesitara.

Claridad en los contratos. Algunos de los diferentes esquemas de servicio incluyen modelos basados en licencias por usuario, licencias compartidas, usos temporales o por períodos fijos, pago por uso y pago por subscripción.

\section{Estándares}

Resulta indispensable que las diferentes plataformas que ofrecen los distintos proveedores de cloud computing estén basadas en estándares que garanticen la compatibilidad y la correcta integración de diferentes servicios de manera simple y rápida.

\section{Disponibilidad}

Cloud computing no evita tener que planear adecuadamente las estrategias de recuperación de desastres y continuidad de servicio. El enlace de red y la energía son otros puntos importantes a tener en cuenta en este aspecto para que no resulten ser un único punto de falla.

\section{Costos}

Puede suceder en ciertas ocasiones que los costos de adoptar un esquema de cloud computing no resulten tan atractivos para aquellas entidades financieras que lo comparen contra los esquemas informáticos tradicionales. La conjunción de los diferentes modelos computacionales y sus costos son los que determinarán si adoptar un modelo de cloud computing podría resultar económicamente mejor que mantener el esquema tradicional. Es necesario tener en cuenta además el costo del almacenamiento. Esto llevará a tomar en consideración además los costos de transferencia de los datos. Nuevamente, la conectividad y energía representan otros elementos importantes a ser evaluados en cuanto a costos.

\section{Conclusiones}

Este trabajo ha tratado los principales aspectos tanto positivos como potencialmente negativos que podrían resultar de la adopción de cloud computing 
para las entidades financieras. Uno de estos puntos es el de los marcos de trabajo y mejores prácticas que se encuentran en desarrollo. Se ha concluido que esta documentación no ha llegado aún a un proceso de maduración tal como para poder basarse en una guía estándar de adopción de cloud computing. Es necesario continuar actualizado en el estudio de estos conceptos de manera de llegar bien preparado a la etapa de maduración de normas y estándares que permitirán a las instituciones financieras abordar de una manera más simple y segura el proceso de adopción de cloud computing. Sin embargo, se presentarán a continuación recomendaciones en cuanto a la consideración de los aspectos más relevantes dentro del análisis de adopción.

\section{Análisis de costos y beneficios}

En primer lugar, es necesario que se realice una evaluación de costos que involucre todos los aspectos mencionados en este trabajo, de manera de conocer con anticipación las ganancias que se presentarán si así resultara.

\section{Análisis de regulaciones}

Se debe realizar un análisis exhaustivo en cuanto a las regulaciones del mercado financiero y legal de cada país en donde residen las entidades financieras.

\section{Selección del modelo de cloud computing}

Una vez que se ha definido que el proyecto es rentable y que cumple con las regulaciones, se debe definir el modelo de cloud computing a adoptar. Se sugiere comenzar con un modelo híbrido, de manera de poder alojar bajo un modelo de cloud privado aquellas aplicaciones más sensibles del negocio, ya sea por cuestiones de disponibilidad; pero lo que es aún más importante, por cuestiones de protección de la información. El modelo de cloud híbrido se completa incorporando a este cloud privado, un modelo de cloud público para alojar aquellos ambientes no productivos.

Cloudbursting. Es un habilitador para el traspaso de información entre el esquema de cloud privado hacia el cloud público. Las aplicaciones son originalmente desplegadas en el cloud privado y a medida que la demanda aumente y no se pueda manejar dentro ese tipo de cloud, se mueven hacia el cloud público. De esta manera las entidades financieras sólo pagarán por los servicios de cloud público cuando los picos de demanda lo requieran.

\section{Evaluación del proveedor}

Con el modelo de cloud necesario y las políticas de nivel de servicio requeridas definidas, las entidades financieras deben evaluar la cantidad de certificaciones que ofrece el proveedor de cloud computing porque éstas garantizarán una mejor integración, ya sea porque disponen de estándares o poseen APIs comunes.

\section{Incorporación de un Cloud Auditor}

Debido a la importancia de los estándares, pero bajo el punto de vista de las regulaciones, un tercer rol entra en juego entre la entidad financiera y su proveedor de cloud computing. El NIST define al auditor de cloud (NIST, 2011) como una 
entidad que realiza exámenes independientes de los controles de servicio de cloud computing con la finalidad de expresar una opinión en base a lo observado. Las auditorías son realizadas para verificar la conformidad con los estándares mediante la revisión de evidencia objetiva.

\section{Administración}

Por último y de igual manera que en los servicios T.I. tradicionales, no se debe descuidar el aspecto de la administración, tanto para la información, accesos de red y servicios. 


\section{Referencias}

Analysys Mason. (2010). Enterprise cloud services: worldwide forecast 2010-2015. Gartner. (2008, 10 14). Gartner Identifies the Top 10 Strategic Technologies for 2009. Retrieved from Newsroom: http://www.gartner.com/newsroom/id/777212 Gartner. (2009, 10 20). Gartner Identifies the Top 10 Strategic Technologies for 2010. Retrieved from Newsroom: http://www.gartner.com/newsroom/id/1210613 Gartner. (2010). Gartner Forecast: "Public Cloud Services, Worldwide and Regions, Industry Sectors, 2009-2014".

Gartner. (2010, 10 19). Gartner Identifies the Top 10 Strategic Technologies for 2011. Retrieved from Newsroom: http://www.gartner.com/it/page.jsp?id=1454221

Gartner. (2011, 10 18). Gartner Identifies the Top 10 Strategic Technologies for 2012. Retrieved from Newsroom: http://www.gartner.com/it/page.jsp?id=1826214

Gartner. (2011). Hype Cycle for Cloud Computing, 2011.

Gartner. (2011). Hype Cycle for Cloud Security, 2011.

Gartner. (2011). Private Cloud Computing: Driving a Strategy.

Global Industry Analysts. (2011). Cloud Computing: A Global Market Report.

IDC. (2010). Cloud Computing in the Insurance Industry.

IDC. (2010). Worldwide and Regional Public IT Cloud Services 2010-2014 Forecast (IDC \#223549) .

IDC. (2011). Best Practices: Cloud Strategies in Practice - NYSE Financial Community Cloud.

IDC. (2011). Best Practices: Software-as-a-Service Case Study - Santander Consumer USA Deploys Salesforce.com for Key Business Unit.

IDC. (2011). Best Practices: Software-as-a-Service Case Study - Sun National Bank Deploys Mobile Banking in the Cloud.

IDC. (2011). Business value of private clouds.

IDC. (2011). Cloud's Beyond The Hype: Positioning for the New Era of Enterprise IT.

IDC. (2011). Perspective: Cloud Computing in Capital Markets.

IDC. (2011). US Financial Institutions and Cloud Adoption - January, 2011 Survey Results. 
Microsoft. (2010). The Economics of the Cloud.

Microsoft. (2011, 5 12). Bank Boosts Cross-Selling by 20 Percent, Speeds Loan Approval, with CRM Solution. Retrieved from Case Studies: http://www.microsoft. com/casestudies/Case_Study_Detail.aspx?CaseStudyID=4000009941

NIST. (2011). Cloud Computing Reference Architecture.

NIST. (2011). The NIST Definition of Cloud Computing (Draft).

Protection of personal data. (2011, 10 10). Retrieved from European Commission Justice: http://ec.europa.eu/justice/data-protection/index_en.htm

Salesforce.com. (2011, 10 10). Commercial Banking. Retrieved from Solutions: http://www.salesforce.com/solutions/financial-services/commercial-banking.jsp

Shashidharan, K. P. (2011, 9 25). Cloud computing for banking. Retrieved from The hindu business line: http://www.thehindubusinessline.com/features/mentor/ article2484918.ece?ref=wl_features

Verstraete, C. (2011). Data and the Cloud.

WinterGreen Research. (2010). Cloud Computing Stack Layers - IaaS PaaS, SaaS Market Strategies, Shares, and Forecasts, Worldwide, 2010-2016. 